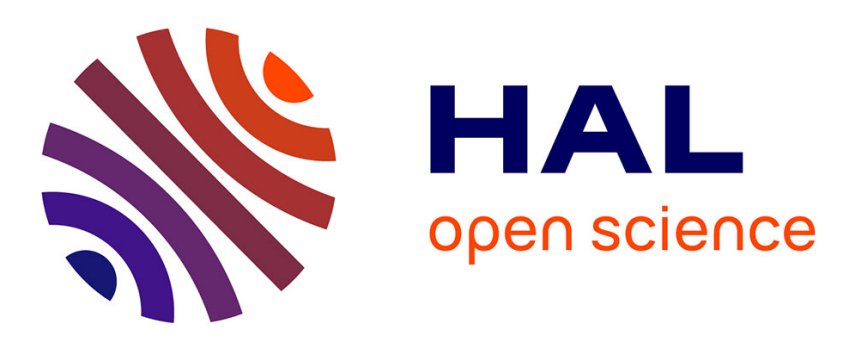

\title{
Exploring the Role of Artifacts in Collective Dance Re-staging
}

\author{
Jean-Philippe Rivière, Sarah Fdili Alaoui, Baptiste Caramiaux, Wendy \\ Mackay
}

\section{- To cite this version:}

Jean-Philippe Rivière, Sarah Fdili Alaoui, Baptiste Caramiaux, Wendy Mackay. Exploring the Role of Artifacts in Collective Dance Re-staging. Proceedings of the ACM on Human-Computer Interaction , 2021, 5 (108), pp.1 - 22. 10.1145/3449182 . hal-03245188

\section{HAL Id: hal-03245188 \\ https://hal.science/hal-03245188}

Submitted on 1 Jun 2021

HAL is a multi-disciplinary open access archive for the deposit and dissemination of scientific research documents, whether they are published or not. The documents may come from teaching and research institutions in France or abroad, or from public or private research centers.
L'archive ouverte pluridisciplinaire HAL, est destinée au dépôt et à la diffusion de documents scientifiques de niveau recherche, publiés ou non, émanant des établissements d'enseignement et de recherche français ou étrangers, des laboratoires publics ou privés. 


\title{
Exploring the Role of Artifacts in Collective Dance Re-staging
}

\author{
JEAN-PHILIPPE RIVIÈRE, Université Paris-Saclay, CNRS, Inria, LISN, France \\ SARAH FDILI ALAOUI, Université Paris-Saclay, CNRS, Inria, LISN, France \\ BAPTISTE CARAMIAUX, Université Paris-Saclay, CNRS, Inria, LISN, France and Sorbonne Université, \\ CNRS, ISIR, France \\ WENDY E. MACKAY, Université Paris-Saclay, CNRS, Inria, LISN, France
}

Preparing a new dance performance involves more than learning individual steps. We are interested in understanding how dancers collaborate as they rehearse a new dance piece, with a particular emphasis on how they use physical and digital artifacts to support this process. We conducted a 12-month longitudinal observational study with a dance company that re-staged a dance piece, taken from the contemporary repertoire and unknown to the dancers. The study focused on the role that artifacts play in shaping the learning of a dance piece. We showed how dancers produced an ecology of artifacts with the aim of analyzing the choreographic ideas behind the dance and sharing them with other learners. We showed that sharing these artifacts was challenging because they are idiosyncratic and embody their creator perspective and vocabulary. We then illustrated how dancers overcome this challenge by compiling artifacts and distributing the learning task among the group in order to create a common knowledge of the piece which improves the learning process. We conclude with design opportunities for technologies supporting long-term dance learning processes.

CCS Concepts: • Human-centered computing $\rightarrow$ Empirical studies in HCI; Empirical studies in collaborative and social computing.

Additional Key Words and Phrases: Dance; Learning; Qualitative Methods; Longitudinal Study; Segmentation; Artifacts

\section{INTRODUCTION}

Learning and performing a new dance choreography requires both skill and practice [21], and offers a fertile ground for studying complex skill acquisition $[4,5]$. Although a number of systems have been designed to teach dance [38], most of them focus on supporting an individual dancer's learning process [1]. However, learning a dance performance is highly collaborative - dancers must not only learn their own parts, but also learn how to interact dynamically with other dancers.

Understanding this process requires a more holistic approach, taking into account the situated nature of cognition, where human knowledge is grounded in its social, physical, and cultural context [44]. We are especially interested in understanding how dancers work together to produce the final performance, with the longer-term goal of designing interactive tools that support the collaborative aspects of dance learning.

Designing for collaborative dance learning requires an in-depth understanding of how dancers collaborate, and this can be completed by observing users in the wild [40]. Within the fields of Computer-Supported Collaborative Learning (CSCL) and Work (CSCW), there are a variety of

Authors' addresses: Jean-Philippe Rivière, Université Paris-Saclay, CNRS, Inria, LISN, Orsay, 91405, France, riviere@lri.fr; Sarah Fdili Alaoui, Université Paris-Saclay, CNRS, Inria, LISN, Orsay, 91405, France, saralaoui@lri.fr; Baptiste Caramiaux, Université Paris-Saclay, CNRS, Inria, LISN, Orsay, 91405, France, Sorbonne Université, CNRS, ISIR, Paris, 75005, France, caramiaux@isir.upmc.fr; Wendy E. Mackay, Université Paris-Saclay, CNRS, Inria, LISN, Orsay, 91405, France, mackay@lri.fr.

(c) 2021 Association for Computing Machinery.

This is the author's version of the work. It is posted here for your personal use. Not for redistribution. The definitive Version of Record was published in Proceedings of the ACM on Human-Computer Interaction, https://doi.org/10.1145/3449182. 
methods to capture collaboration in the wild. A popular approach is to examine the artifacts produced by the group to understand the relationships between the different protagonists [46] Recently, this has been successfully applied to creative practice, where digital and physical artifacts served to analyze collaborations between creators and performers in music and dance [26]. We follow the same direction, using artifacts as a lens into the collaborative practices of learning dance. In this paper, we address the following research questions: How do dancers learn a dance piece in a group? What are the different ways in which dancers interact with digital and physical artifacts in collaboration?

We collaborated with a dance company working on the re-staging of a professional dance piece from the contemporary dance repertoire. In this context, re-staging means that the dancers of the company were required to learn and perform an existing dance piece unknown to them, as closely as possible to the choreographer's original idea. During our collaboration, we ran a longitudinal field study where we investigated how the company's dancers interact with both digital and physical artifacts to collectively learn and rehearse the dance piece. We introduced the dancers to an existing interactive system designed to support dance learning, called MoveOn. We observed in our study how MoveOn was used and integrated into their learning process alongside the other artifacts.

We show that participants created and appropriated an ensemble of heterogeneous complementary artifacts that help them to decompose and analyze the choreographic ideas underlying the dance. However, we show that sharing these artifacts is challenging because they embody their creator's idiosyncratic perspective and vocabulary. Our results illustrate how participants overcame these challenges by building a common knowledge of these artifacts and by distributing expertise among the group.

We discuss our results in light of the limitations of current approaches and provide guidelines for future technologies for supporting dance learning. We also emphasize the inherent complexity of collaborative dance learning.

The paper proceeds as follows: we first present the literature on different approaches and interactive systems designed to support dance learning. We then introduce the context of the dance piece and MoveOn. Next, we introduce our methodology and describe the results of our observational study. Finally, we discuss our results and provide design guidelines to build dance supporting tools that can be used in real-life dance learning and rehearsal processes.

\section{RELATED WORK}

We are interested in how artifacts mediate the learning process of a group of dancers. We first introduce the different research in the CSCL and CSCW fields that investigate how artifacts mediate collaborative learning. We then review how physical and digital artifacts intervene in the specific context of dance learning and practice. Throughout the review of these related works, we discuss the gap between real-life dance practice and how existing systems that support dance learning are designed and evaluated.

\subsection{Physical and digital artifacts in collaborative practice}

The CSCL literature studies how computers mediate collaborative learning processes. As stated by Stahl [46], knowledge building is mediated by the production of knowledge objects or learning artifacts. Learning artifacts are lasting, durable, and public objects created by the learner for a specific use [42]. They provide concrete evidence and a basis for evaluating knowledge building and interactions within a group [46, 47]. Bødker and Klokmose [6] showed that the relationship between artifacts evolves dynamically over time. This suggests the importance of considering the ecology of artifacts over a long period. 
While the CSCL and CSCW literature focuses mainly on cognitive learning tasks (for example mathematical reasoning), there is much to be done in studying motor learning and how artifacts intervene in this process. Such a gap in the literature is perhaps due to the assumption that practitioners might produce fewer artifacts when learning through the body. However, two recent studies [14,26] identified, in the specific case of dance, the various artifacts that dancers and choreographers create to document their practice.

2.1.1 The role of physical artifacts in dance practice. In the dance studio, practitioners produce physical artifacts such as notes, sketches, or notations of their dance ideas, performances or choreographic structures [33, 48]. As depicted in [32], in a dance creation process, dancers and choreographers produce handwritten notes, which can, in turn, be exploited by researchers to get a better understanding of the dance-making process. In the same context, Ciolfi Felice [13] showed that choreographers use drawings, text, diagrams, and videos primarily during the preparation of a choreography, but rarely when reflecting upon the piece. She describes that both choreographers and dancers use artifacts to transmit choreographic ideas. However, these two studies do not investigate the use of artifacts in the specific process of collaborative dance learning.

In the dance studio, physical artifacts are produced by both the teacher and the learners. Artifacts act as objects that preserve a common memory across the group and can be studied to reveal the relationship inside a group. These artifacts are usually part of an ecology which refers to the idea that they are connected and influence each other [30], and should not be considered in isolation.

2.1.2 The role of digital artifacts in dance practice. Nielsen and Bødker [36] show how digital artifacts can be used in coordination with other physical artifacts such as paper notes or tools. In a dance setting, practitioners also produce digital artifacts to support dance transmission, production, annotation, and archive [34]. Videos are among the most common type of digital artifacts used in dance studios. Today, dancers and choreographers video record their dance performances to share, transmit, or archive them. For much of the modern and contemporary dance repertoire, video recordings act as a reference that allow dancers to learn repertoire pieces.

Recent studies in HCI have investigated the combination of digital and physical artifacts to inform the design of interactive systems supporting choreographic writing [15] or to enhance kinaesthetic creativity in dance improvisation [27]. In a recent paper, Hsueh et al. [26] investigated how choreographers interact with the digital and physical artifacts that they create. They identified various approaches ranging from sculpting to layering to remixing these materials. The authors also identified the different roles that choreographers take to collaborate with dancers. They found that creators navigate the interactions with their artifacts and distribute the roles among collaborators fluidly. They argue that this fluidity observed in the real-world opens myriad possibilities and should be accounted for the design of systems supporting creativity. Similarly, the study of artifacts in collaborative dance learning contexts can shape our conception of tools supporting both the learners and the teachers.

\subsection{Annotation systems in dance learning}

Annotation tools have been successfully introduced into real-life learning contexts. These systems are used to document and preserve the choreographic process [9], to promote communication between a teacher and dancers [43], and to support dance learning [19]. They not only present interesting archival value but also have the potential to enhance dance learning through collaborative text and annotations [20]. The Choreographer's notebook designed by Singh et al. [43] is a web-based application that allows choreographers and dancers to collaborate in the analysis and annotation of rehearsal videos. The authors ran a field-study of this system in three dance productions [11], and argue that such systems can increase efficiency of rehearsal time, support 
learning, and enable online communication between the dancers and the choreographer. However, they found that dancers do not use this tool to comment on each other's performance and that choreographers make most of the comments, suggesting that there is a hierarchy between dancers and choreographers. We want to investigate further this phenomenon and the roles of the different practitioners in the learning process.

dos Santos et al. [19] present a video annotation tool where dance teachers can write comments or use predefined labels to assess a dance performance, but only in a one-to-one setting (one teacher to one dancer). Recently introduced, DanceNote, created by the company La fabrique de la danse, is a web tool that encourages choreographers and dancers to share, annotate, and learn from video content ${ }^{1}$. This tool has been used in real dance learning contexts; however, there is no study nor evaluation of the use of this tool by dancers. Rivière et al. [39] present MoveOn, a technology probe designed to support dance learning. MoveOn takes the form of a video media player, where dancers can segment a video into repeatable dance clips. The authors tested this tool in two structured observations [24], close to real dance practices, and suggest that it is valuable for learning dance video excerpts. These two studies take place over a short learning period, where dancers do not communicate nor collaborate with each other. We aim to utilize the same tool (MoveOn), but in a real collaborative dance task, where it will be used among other digital artifacts.

Finally, Raheb et al. [38] propose an extensive literature review of interactive systems that support dance learning. The majority of the systems presented follow the same workflow: the system presents a "correct movement" that the student needs to reproduce while the system provides feedback. These types of systems are often designed for one singular dancer and are usually assessed through isolated tasks in a laboratory $[1,2,12]$. This calls for more research that leverages the collaborative nature of dance learning and systems such as annotation tools.

\subsection{Theories on embodied practices}

Multiple theories provide insights for the design and study of digital artifacts that support embodied practices. These theories are important for structuring our methodology and our understanding of the artifacts created by the group.

2.3.1 Situated and embodied cognition. According to the theory of situated cognition presented by Solomon [44], all human knowledge is situated in a social, physical, and cultural context. Therefore, studying how human beings learn involves broadening our perspective on the context in which it occurs. Similarly, the theory of distributed cognition widens our perspective on cognition to include interactions between people and with resources and materials in the environment [28, 41]. Artifacts can convey, store, and distribute information among individuals and therefore support practice and collaboration [25].

According to Varela and colleagues [50], in their enactive approach, cognition is not only situated but also embodied, which means that physical characteristics and configurations are constitutive to cognition. Artifacts are crucial to understanding how we embody the world in which we live. For example, Hutchins [29] studied coordination between Navy personnel in navigation tasks and showed how cognition is distributed across people and tools, and how artifacts are crucial for the execution of a collaborative task.

2.3.2 Collaborative physical thinking. In the specific case of dance, in addition to relying on groupproduced and self-produced (physical or digital) artifacts, dancers use their bodies to think, learn, and transmit their knowledge $[17,18]$. They constantly adapt their movement to the environment and develop a Kinaesthetic intelligence and an acute capacity for physical thinking [18]. Such

$\overline{{ }^{1} \mathrm{http}: / / w w w . l a f a b r i q u e d e l a d a n s e . f r / d a n c e n o t e / ~}$ 
embodied capacities are collective as dancers are usually connected to each other on stage and their cognition is distributed across their brains and bodies [10,49]. While there are numerous systems designed to support physical thinking and kinaesthetic intelligence in dance, most of them are designed regardless of the collaborative aspect of dance and are assessed individually, for one body isolated from the rest of the group. Designing for dance learning involves a better understanding of the collaborative and distributed aspect of dance and the environment in which it occurs. Artifacts are a medium to study the acquisition of knowledge by the dancers, as well as the collaboration between dancers and teachers, in real-life dance learning tasks.

\section{CONTEXT}

Collaborative dance learning is a complex process grounded in a social, physical, and cultural environment. Designing interactive systems for such contexts requires diving into the reality of the dance studio. We had the opportunity to collaborate with a dance company called De l'air dans l'art (consisting of 12 dancers, a teacher, and a company director), on the re-staging of Frame(d). This piece is a fusion of two existing dance pieces $\left(B a b e l^{2} \& M y t h^{3}\right)$ originally choreographed for the Eastman company ${ }^{4}$. Frame(d) requires high skill level and group coordination which can only be acquired through multiples rehearsal sessions.

The process of re-staging Frame(d) started in October 2018 with a five-day dance workshop and ended with the final performance in February 2020. Between October 2018 and February 2020, the rehearsal director led 27 sessions (111 hours), and the company director led eight sessions (32 hours), for a total of 35 rehearsal sessions (143 hours). Although not paid by the company, the 12 dancers were strongly engaged in the project and spent much of their free time rehearsing together. Figure 1 shows a timeline of the project.

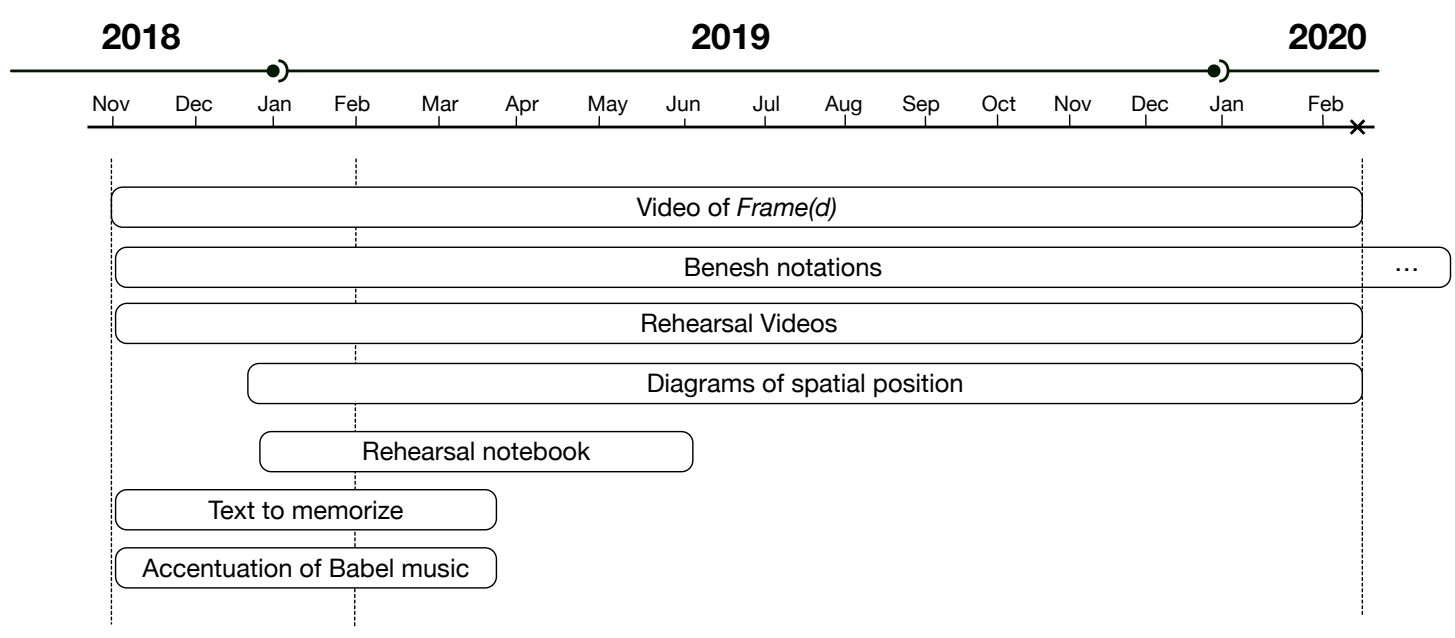

\section{First rehearsal Study started}

Fig. 1. Project timeline. The company deployed and maintained different artifacts (rectangles) during all the re-staging process.

\footnotetext{
${ }^{2}$ Babel: http://www.east-man.be/en/14/20/ choreographed by Sidi Larbi Cherkaoui and Damien Jalet

${ }^{3}$ Myth: http://www.east-man.be/en/14/31/ choreographed by Sidi Larbi Cherkaoui.

${ }^{4}$ http://www.east-man.be/
} 


\section{1 $\operatorname{Frame}(d)$}

Frame (d) is a 20 minutes dance choreography for 12 dancers, composed of 9 parts. Dancers refer to them as: 1) the line, 2) trios, 3) the bus, 4) the maze, 5) the leaves, 6) the technology, 7) the $V$, 8) the trio, and 9) the pyramid. Each part of the piece presents particular characteristics that we describe below.

When the auditorium gets dark, the dancers enter the stage one after the other, line up, and sit on their knees facing the public, as shown in Fig. 2a. When the music starts, they collectively perform a fast sequence of movements, while moving forward and standing up. In the second part, dancers position themselves in trios on a diagonal and execute synchronized movements. In the third part, the group forms one trio, one quartet, and one quintet. Then they regroup in the middle of the scene and form a crowd, where, in a perpetual flow, they push each other to be in the foreground. This part is called the maze (Fig. $2 \mathrm{~b}$ ) because one after the other, they leave the crowd, following a squared path. Part 1 to part 4 is characterized by rapid and precise movements following the rhythm of percussive music, played with claves ${ }^{5}$ and drums. At the end of part 4 , the music stops and the dancers fall to the ground.

Part 5 is a transition from percussive to a calmer and more ambient music. One of the dancers stands while the rest lie on the floor. The standing dancer wanders among the leaves (Fig. 2c) that gradually begin to move. In part 6, called the technology, electronic music gradually rises. The dancers perform robotic movements that are jerky and synchronized with the music. In part 7, one dancer walks in the foreground to declaim a text (the text is a transcript of a Ted talk given by the neuroscientist Vilayanur Ramachandran ${ }^{6}$ ). Gradually, the dancers take a V-shaped formation (Fig. 2d) where they all stand and perform the same movements with their arms. After 2 minutes - halfway through the text - the speech is echoed by all the dancers; they declaim it all together while moving, then start the second trio (Fig. 2e) while only three dancers stay on stage. Two of the dancers are on the floor and give their hand to a third who stands (their hands will stay bound during the trio). Finally, in the last part, the rest of the dancers join the trio and form a pyramid of bodies. The soloist climbs this pyramid while trying to reach the sky (representing the Tower of Babel) (Fig. 2f).

\subsection{Integration of a dance learning support system}

In collaboration with the company director, we introduced an existing system designed to support dance learning from videos, called MoveOn [39], shown in Fig. 3. Because re-staging Frame(d) involves learning from several videos, the company director saw this tool as an opportunity to facilitate the dancers' interaction with the videos. Moreover, the dancers of the company were keen to explore the use of new technologies in their learning process.

Initially, our goal was to study how dancers use MoveOn in a real context. However, we quickly realized that in real rehearsals, dancers use an ensemble of tools that they compose together. Thus, the study observed the ways in which MoveOn facilitates collaborative learning in interaction with a larger panel of tools and artifacts that dancers create and share.

MoveOn is a web-based technology (Node.js ${ }^{7}$ ) designed for tablets and laptops that lets users decompose a video into multiple segments and keep a trace of this decomposition. Each segment is an interactive object associated with a part of a video. When a segment is clicked, its associated video is looped at a defined speed. A user can create a segment by defining a starting point and an ending point in a video, annotate the segment, and define a speed using a drop-down menu.

\footnotetext{
${ }^{5}$ Percussion instrument

${ }^{6}$ https://www.ted.com/talks/vilayanur_ramachandran_the_neurons_that_shaped_civilization

${ }^{7}$ https://nodejs.org/en/
} 

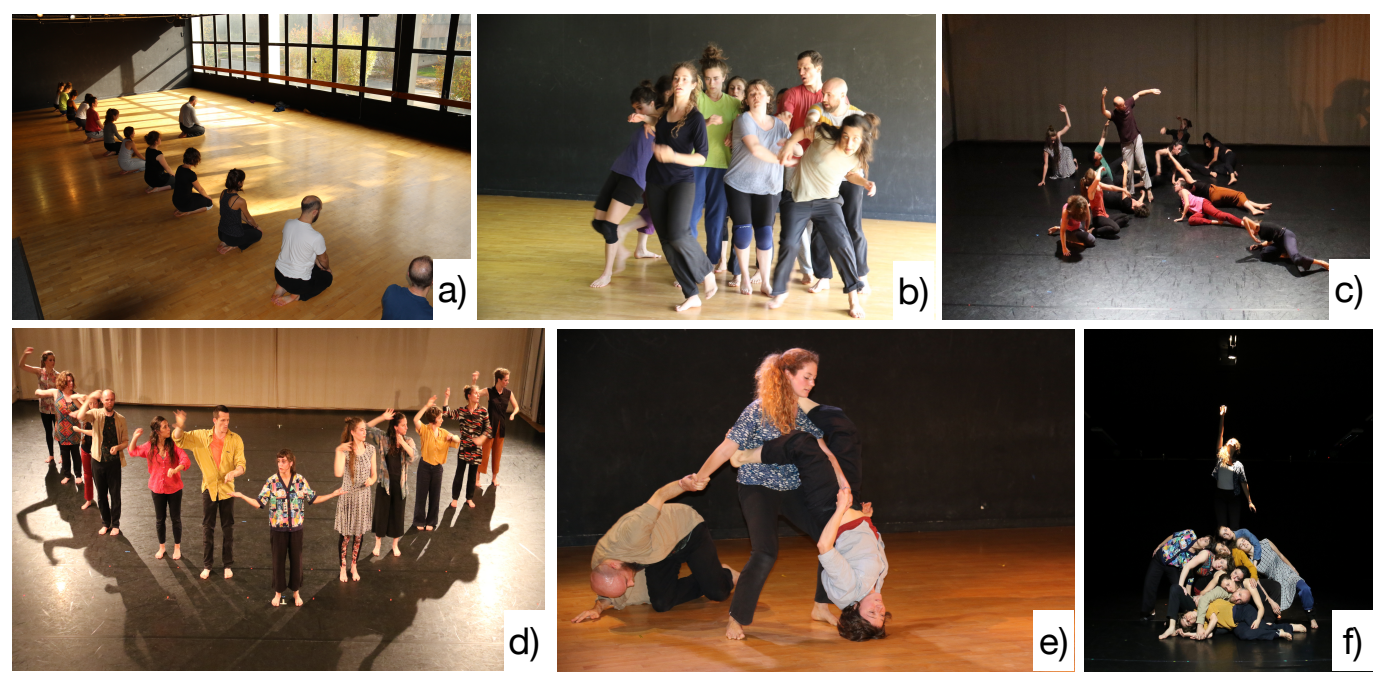

Fig. 2. Different formations in Frame(d): a) line, b) maze, c) leaves d) V, e) trio, and f) pyramid.

The visual representation of a segment embeds two properties: its starting point in the video (represented by its left margin) and its duration (represented by its length). MoveOn saves all the segments created by the users, which can then be shared and reused by other dancers to navigate inside a video.

\section{METHODOLOGY}

In this study, we observed the dancers, the director of the company, and the rehearsal director in their real-life re-staging process. We gathered the physical and digital artifacts created by the company and observed their use and evolution throughout the re-staging. These physical artifacts and technologies support the knowledge building of the learners and provide us with a tangible trace of the learning process [45]. We gathered and studied them in order to better understand the underlying mechanisms of collaborative dance learning.

It is important to note that, prior to our collaboration, the dancers rehearsed with their teacher for 32 hours (in 2018). The first four parts of the piece were deeply analyzed by the dancers, and a primary version of the artifacts related to these parts was created. We intervened in the re-staging process after the creation of the artifacts. We witnessed most of the rehearsal process as we spent 111 hours with the dancers in the studio and were able to trace the lifespan of all the artifacts.

\subsection{Participants}

The 12 dancers (10 females, 2 males) were selected for Frame $(d)$ by the company director through an audition process Those selected were experienced contemporary dancers with more than 10 years of practice. Seven of them were professional dancers and dance teachers (Dancers 2, 4, 8, 9, 10, $11 \& 12$ ). Of the 7 professional dancers, 2 of them (Dancers 4 \& 12) were Benesh notators. Benesh notation is a codified system of writing dance scores for a given choreography. The five remaining dancers were not professional dancers but highly skilled practitioners.

The company director was a dance teacher with more than 40 years of experience in dance pedagogy, and was in charge of the administrative, financial support and coordination of rehearsals. 


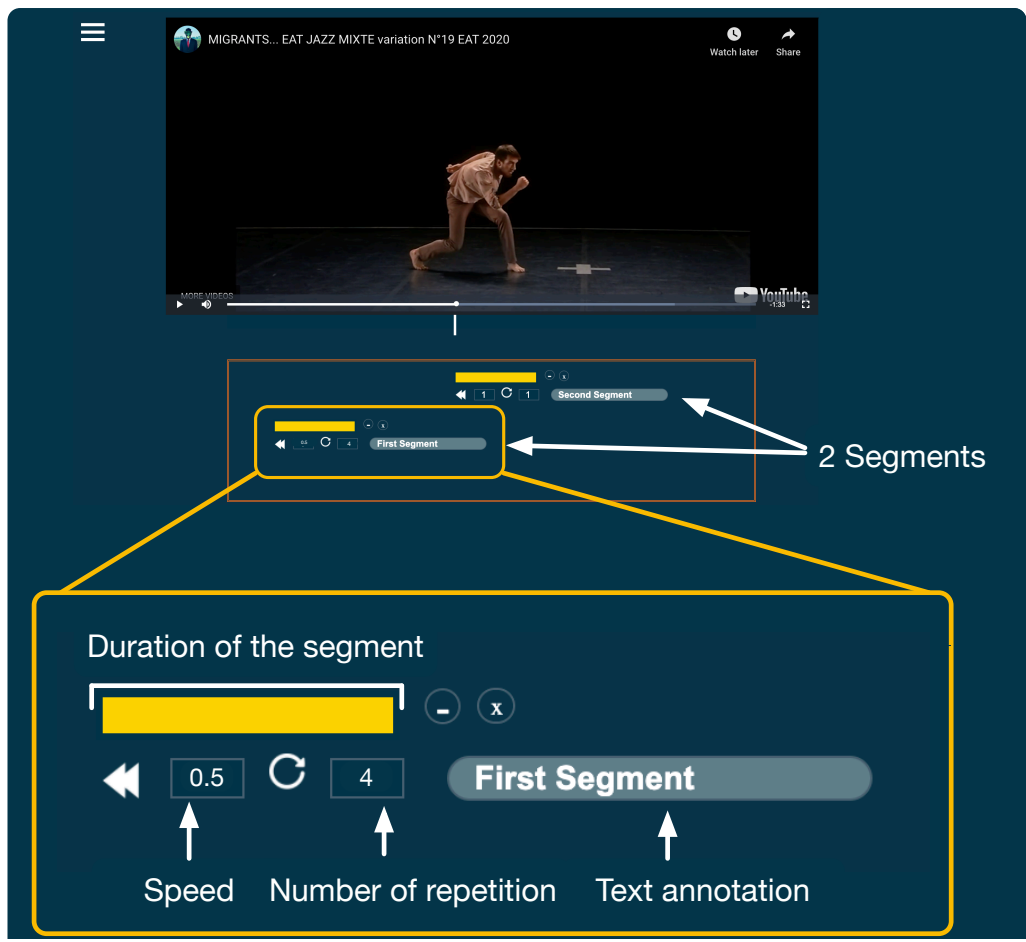

Fig. 3. The interface of MoveOn. The upper half represent a dance video and the lower half a segment history (orange box) which includes two segments (two yellow bars). For each segment, a user can annotate the segment and change its speed and its number of repetition. When a user selects a segment, its associated video part is repeated at a defined speed.

The Eastman company allowed the reproduction of Frame(d) only if the piece was reproduced identically. To fulfill this condition, the company recruited a rehearsal director. The rehearsal director was a professional dancer, from the Eastman company, with 40 years of experience in dance. He already performed Frame(d) on stage, and his role was to transmit the choreography to the dancers and to direct the rehearsals. He directed half of the rehearsals in the presence of the company director, while the other half was directed by the company director alone. In what follows, we refer to dancers 1-12 as D1-D12, to the rehearsal director as $\mathrm{RD}$, and to the director of the company as $\mathrm{CD}$.

\subsection{Procedure}

We conducted a longitudinal study with the dancers and the rehearsal director where we performed group discussions, interviews, and observations. During each rehearsal, we brought three to six tablets and connected them to MoveOn through an internet browser. During the first rehearsal with the dancers, we presented the technology and its features (creation of a segment, navigation between videos, etc.). We invited the dancers and the rehearsal director to use MoveOn instead of their usual media player, cautious not to impose the use of the technology. We observed how the dancers and the rehearsal director used digital and physical artifacts as well as MoveOn to support transmission, learning, and rehearsal of the dance. 
We conducted two group discussions with the dancers and the director of the company. We used a variation of Flanagan's Critical Incident Technique [23] introduced by Mackay [35] for HCI. In the first group discussion (three months after the beginning of the study), we asked the participants to explain their use of technology in the dance studio, and how it shapes their learning. At the end of the study, we performed a second group discussion with the company. Step by step, we reviewed all the artifacts created and used by the company, to discover by whom, where, why, and when they were created, and how they were integrated into the learning process.

We performed an interview with the rehearsal director on the same topic. The interview lasted approximately 60 minutes and took place via Skype. Finally, we sent a form to each participant where we asked them to report on each artifact, if they used it, annotated it, shared it, when they used it, and if it was useful to them. During and after the study, the first author was in close contact with all the participants.

\subsection{Data collection}

Being in the field and attending the rehearsal allowed us to collect a copy of each artifact shared and maintained by the dancers. This includes their handwritten notes, textual representations, sketches, musical scores, diagrams, Benesh notations, rehearsal videos, and tutorial videos. We present these artifacts in Table. 1, and for each one we indicate who created it, and the number of dancers who used and shared it. We kept a research notebook of our observations where we documented the learning process with photos and notes. Additionally, we tracked the modifications that were made to MoveOn with Git, a free and open-source Version Control System. During all the interviews, we recorded audio and video and took hand-written notes.

\subsection{Data analysis}

For each artifact, we counted how many dancers used it, appropriated and shared it, and for how long. We compared the artifacts corresponding to the same part of the piece to understand the representations used. When a point was unclear, we asked their creator for a detailed explanation.

To analyze the group discussions and interviews, we first transcribed audio and video data and anonymized all interviews. We then performed a thematic analysis [7] to extract themes related to the creation and use of the digital and physical artifacts. Thematic analysis is a well established method in psychology and HCI research to analyze qualitative data. It involves researchers actively identifying themes in the interview data. Two of the authors analyzed the data allowing for collaborative discussion and cross-checking of participants' stories. The first analyst is the main investigator, in contact with the dancers during all the rehearsals. The second analyst is an HCI researcher, expert in dance, and familiar with the issues faced by dancers. We identified concepts using open coding and grouped them into themes. We followed a bottom-up approach where we actively defined and named themes from participants' stories.

\section{RESULTS}

In this study, we observed that learning dance relies not only on one tool but on an ensemble of heterogeneous and complementary artifacts that dancers create, appropriate, and share (see Table 1). We describe hereafter the purpose of the artifacts created which is to help decompose movement and focus on specific aspects of the choreography (the rhythm, position in space, etc.). However, we illustrate the challenges around the readability and accessibility of these artifacts that are due to the fact that they highly embody their creators' perspective, expertise, and personal vocabularies. Finally, we show how the participants overcome these challenges by compiling and appropriating the artifacts and by distributing the knowledge among each other. 


\begin{tabular}{|l|l|l|l|}
\hline Artifacts & Created by & Used & Shared \\
\hline Tutorial videos & RD, D2, D4, D5, D9, D10 & 9 & 5 \\
\hline Rehearsal videos & RD, CD & 12 & 0 \\
\hline Video of Frame $(d)$ & Eastman (dance company) & 12 & - \\
\hline Benesh notations & D4, D12 & 2 & 2 \\
\hline Rehearsal notebook & CD, D3 & 10 & 0 \\
\hline Textual representation of Technology & RD & 8 & 1 \\
\hline Accentuation of Babel music & RD & 10 & 1 \\
\hline Rhythmic score & D4 & 8 & 1 \\
\hline Diagrams of spatial position & D5 & 7 & 2 \\
\hline Text to memorize (part 7) & Eastman (dance company) & 8 & 3 \\
\hline
\end{tabular}

Table 1. All the artifacts created, used, and shared by the dancers, the rehearsal director, and the company director

\subsection{Artifacts are used to decompose or simplify the choreography}

The dancers and RD created several physical and digital artifacts related to different parts of the piece. Each artifact takes a different form such as videos, texts, diagrams, scores, or notations, among others. We found that artifacts help decompose movement and focus on specific aspects of the choreography (the rhythm, position in space, etc.). Moreover, we found that the information represented in the artifacts used is complementary.

5.1.1 Videos represent movement in dance. $\mathrm{RD}$ explained that videos are convenient to represent the "movement of the body itself". They are the primary type of (digital) artifact that the dancers used in the studio during the learning process for various purposes:

I used them [the videos] at the beginning to specify the movements and the counts (with slowed down music). In the middle, before rehearsals to refresh my memory. But not at the end, because I already embodied the rhythm and these videos made me doubt. (D2)

Besides the video of Frame $(d)$, the dancers and the rehearsal director also filmed and shared numerous other videos. RD produced and shared video tutorials where he marked the movement (i.e. performs it in less than a complete manner). Marking movement is a technique that allows the dancers to simplify the choreography, by isolating elements of it such as upper body movements, rhythm, or accentuation [32]. Most of the dancers (10/11) found these video tutorials useful to understand the difficult parts of the dance.

5.1.2 MoveOn help decompose movement. MoveOn was used to decompose videos and to focus on its parts. Throughout the months during which the dancers used it, they acquired a large expertise in editing their videos with it. For example, D6, D3, and D2 relied highly on MoveOn to learn their trios (part 2). Indeed, D6, D3, and D2 expressed that the tool was useful for these specific trios where the rehearsal director's knowledge was lacking.

It [MoveOn] was very useful for parts that $R D$ did not teach to us. The options of slowing down and selecting a very short extract, really helped me to break down the steps and to unravel the trio with D6 and D3. It is a task that usually requires a lot of time and effort and MoveOn simplified it. (D2)

MoveOn has also been appropriated by $\mathrm{RD}$ to decompose the music. RD created segments according to the music rather than the video. He tweaked the speed of the music to slow down the dance. He reports that: 
I use it more like a musical software. I know the choreography and now, I prefer to rely on the music and not on the video. What I need is to start at a precise point in the video, where the music begins. It is much more precise than my other tools actually. (RD)

While most dancers found MoveOn useful, they deplored that it was not present at the beginning. As explained by D6, "It would have been perfect to have the tool at the beginning of the learning, it came a little late" (D6). Indeed, MoveOn allows decomposing movements into chunks which can be useful in the early phase of analyzing the dance. However, when we introduced MoveOn, parts of the choreography were already analyzed. Thus, dancers used it to analyze mostly the parts of the dance that were not yet known to them. This is illustrated in Fig. 4 where three dancers use MoveOn to decode their trio.
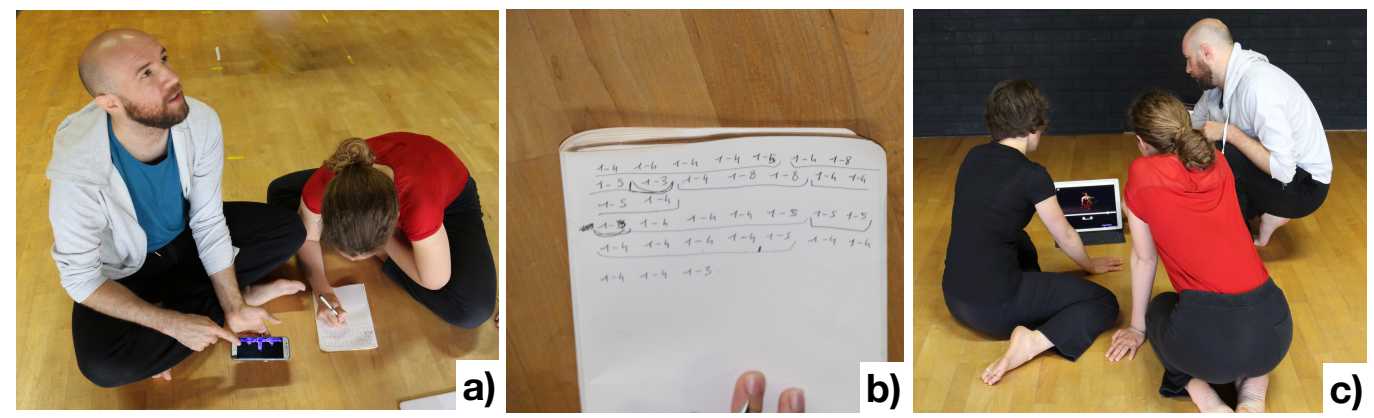

Fig. 4. Pictures of D4, D10, and D12 working on their trios. The picture a) represents D5 and D10 counting the steps from videos and annotating it in their notebook (b). In the picture c), the three dancers watch their part with MoveOn.

5.1.3 Physical artifacts direct focus on key aspects of the choreography. In addition to digital artifacts, the participants produced physical artifacts to focus on specific elements of the piece such as the rhythm, the choreographic cues, or the position in space.

At the beginning of the re-staging, RD shared with the group two documents that he created when he was learning Frame(d), eight years ago. The first document is a text representation of part 6 of the choreography (Fig. 6a). RD shared this document to help the dancers better understand what he calls the choreographic cues:

There is a lot of cues, inside each scene. Cues are reference points that indicate when to begin or end a movement. It's usually musical or visual, either you listen to the music or you see a landmark. These are indications of when you should start or stop your movements.

Complementing RD's document, D5 created 7 diagrams that represent each part of the piece (an example of D5's diagram is shown in Fig. 6b). In these diagrams, he depicted the position of each dancer in space in order to "structure the dance phrases as a whole" (D5) and to see where "the interactions are located" (D5).

$\mathrm{RD}$ provided a second document from his archives that represents the accentuation of the music of part 1 (shown in Fig. 5a). In this document, the red numbers highlight the rhythm and the accent of the music, where movements must be percussive. $\mathrm{RD}$ reported that this document results from a problem he encountered to embody the rhythm. His strategy was to write down the accents of the music on paper and to memorize it: 


\section{a) Transcription of the rhythm} (Created by the Rehearsal Director)

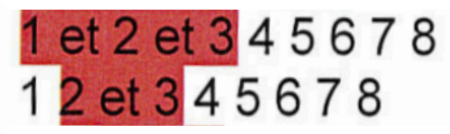

b) Transcription of the rhythm

(Created by D4)

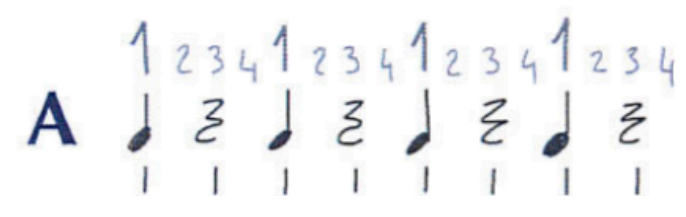

Fig. 5. Dancers have their idiosyncratic representation. RD and D4 created two different artifacts to represent the rhythm and accentuation of the music of, respectively, part 1 and part 7 of the piece. On the left, $\mathrm{RD}$ represented the rhythm in text form where accentuations are colored in red. On the right, D4 represented the rhythm in the form of a musical score where accentuations are indicated by large numbers.

Several kinds of learning exist, either you can memorize it musically or you can visualize it. This document was handy for that. Here, I just isolated the musical side in terms of pause and movement, with colors and punctuation. (RD)

D4 (the Benesh notator) also created a document to represent the rhythm of the music in part 6. D4 decided to represent the rhythm through a formal musical score for its unambiguity (Fig. 5b).

5.1.4 Artifacts provide complementary views of the piece. In Fig. 6a) and Fig. 6b), both documents created by RD and D5 refer to the same part in the piece, but represent different, yet complementary information. On the left, RD used a text to explain dancers' position, while on the right, D5 proposed a diagrammatic representation.

While the form and language used to represent information vary from an artifact to another, the group considers each artifact as presenting an indispensable, mutually complementary view of the piece. Half of the dancers (6/11) found RD's text representation useful to understand choreographic cues, more than half of the dancers (8/11) found D5's diagrams useful for clarifying the space, $(8 / 11)$ and (9/10) dancers found respectively D4's musical score and RD's representation of the accentuation was useful for memorizing the rhythm.

$\mathrm{RD}$ noted that these diverse representations of the same information are important for creating a complementary view of the dance:

I have the feeling that we all sharpened one thing, and all learning angles are complementary at different levels, we have different learning strategies. (RD)

\subsection{The idiosyncratic nature of the artifacts limits accessibility}

We observed many challenges to the appropriation of artifacts by participants who were not their original creators. First of all, each artifact represents the piece through its creator's own perspective and vocabulary. These personal representations sometimes collide with other learners' ideas of the choreography. For example, D11 stated that she would have described differently the diagrams produced by D5:

I did not use D5's document [diagrams] because he oriented it in one way, and I would have oriented it in the opposite way. Because we think and work differently. (D11) 
a) Textual representation of Technology

(Created by the Rehearsal director)

$[\ldots]$

Formation of duos in accumulation :

The first duo is formed at $1 \times 8$ (musical marker change), at $2 \times 8$ the second duo, at $3 \times 8$ the duo 3 and 4 , at $4 \times 8$ the 5 th duo, at $5 \times 8$ the duo 6 . $[\ldots]$

\section{b) Diagram of Technology}

(Created by D5)

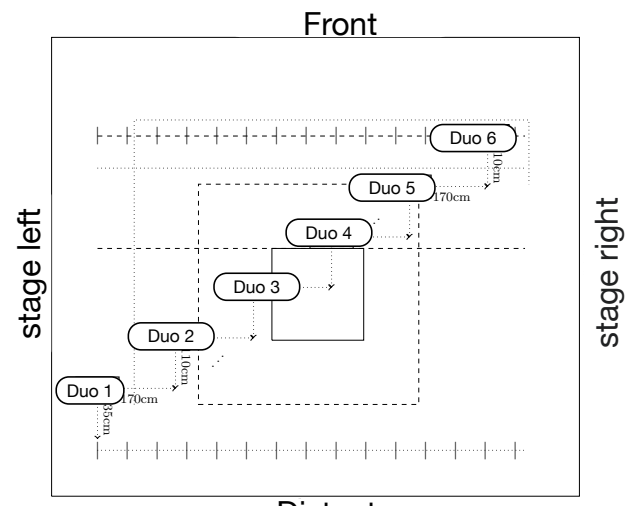

Distant

Fig. 6. Two pictures related to the first part of the piece. On the left, a textual representation created by the rehearsal director indicating when each duo is formed. On the right, a diagram created by $D 5$ represents a snapshot of each duo's position. These two artifacts represent complementary information: time (when duos are formed) and space (where duos are placed).

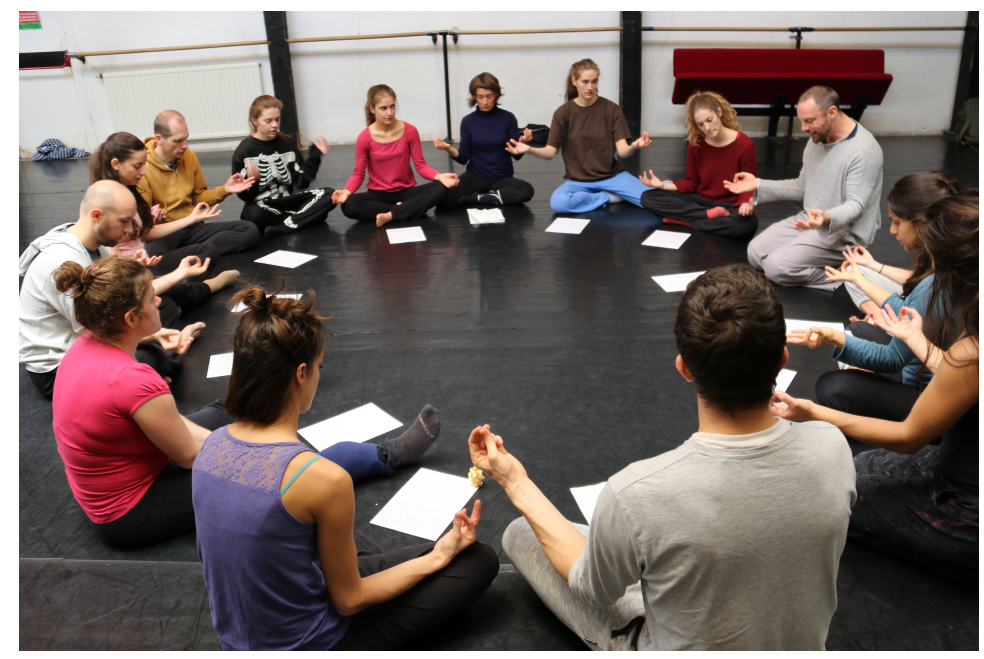

Fig. 7. The dancers and the rehearsal director memorize the text recited in the sixth part.

D11 did not want to use D5's diagrams because there was a mismatch between how D5 represented the diagram and how she would have done it. The direction of the scene was "awkward" to her and did not conform to her mental model of the scene.

Secondly, the lack of a common vocabulary also limits access to the artifacts. D2 reflected on how such issues arose in the rehearsal notebook. 
If we make a common document, how can we express it so that it speaks to every dancer, but at the same time we need this common document because sometimes we forget these corrections which are useful to all of us. (D2)

Although sharing artifacts is considered valuable in order to build the group's common understanding of the piece, such collective action requires communication and "agreement upon the vocabulary used" among participants (D11). RD argued that producing videos was a way to overcome the limitations due to personal vocabularies, because according to him the body is "the common language for dancers" (RD), and videos are the most "readable" artifacts for dancers.

Finally, we observed that expertise limits the access to artifacts. Some artifacts follow precise rules of composition and require a certain expertise, which can elude some learners. For example, D4 created a musical score (Fig. 5b) which requires training in musical theory in order to read it. Three dancers reported that they cannot use it. Another example is Benesh notations, a highly structured form of dance notation that requires extensive training to read or write it. D4 and D12 are two Benesh notators who notated the whole piece to create a sustainable archive of it (an example of the Benesh notation can be seen in Fig. 8). Their notations are considered valuable but not accessible to other dancers. D1 stated: "You have to be a notator to read Benesh notation, and you can't access it otherwise."

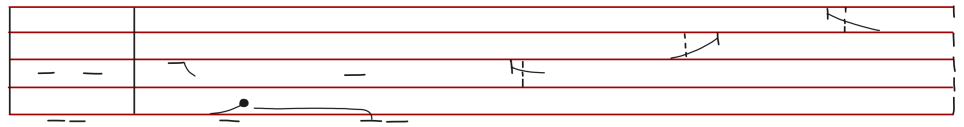

Fig. 8. An example of a Benesh score from part 4, created jointly by D4 and D12.

\subsection{Overcoming challenges of sharing artifacts through compilations}

We observed that compiling documents and videos allow participants to overcome the challenges around the accessibility of the artifacts.

5.3.1 Compilations create an ecology of artifacts. Throughout the whole learning process, participants compiled the artifacts that they consider useful to the group. We illustrate the ensemble of artifacts around Frame (d) in Fig. 10. This figure shows that participants created a system where heterogeneous digital and physical artifacts (orange rectangles) co-exist. Artifacts are grouped through compilation platforms (grey rectangles) which provide the same information to all the participants. Through these platforms, the participants have access to the artifacts produced by the other participants and are free to use the artifacts they want or need. They pick among all the artifacts created by the group to appropriate and adjust their personal learning practice. Thus, dancers create their own network of artifacts which can be understood as an ecology of artifacts, following Vyas and Dix's definition [51].

5.3.2 Compilations facilitate dancers' access to dance knowledge. In the early stage of re-staging, the participants mainly used emails to share their documents and videos. However, limitations rapidly arise: internet access is usually limited in dance studios and dancers do not necessarily bring their laptops to the rehearsals. Under the impulse of CD, D3 created the Rehearsal Notebook, a document that compiles all documents created among the group. $\mathrm{CD}$ printed twelve versions of this rehearsal notebook and distributed it to all dancers. The first version of this document regrouped:

- the text learned in the part 7,

- the musical score wrote by RD and D4 (Fig. 5a, 5b), 
- a diagram of part 6 (Fig. 6b),

- a textual representation of part 6 (Fig. 6a); and

- the schedule of all future rehearsals planned by CD.

This rehearsal notebook provide a unique source of information to everyone, as D3 explains:

Each time we had a lot of comments and a lot of things to say, a lot of things to add. We needed to condense all of this in a handy document. (D3)

The dancers appropriated this document for their personal use, as explained by D2: "At first, everyone has the same notebook, and then everyone built on it.". For example, D12 cut out and integrated part of the rehearsal notebook into her own dance diary (depicted in Fig. 9). We observed that five dancers annotated their version of the rehearsal notebook, taking personal notes on the different sections.

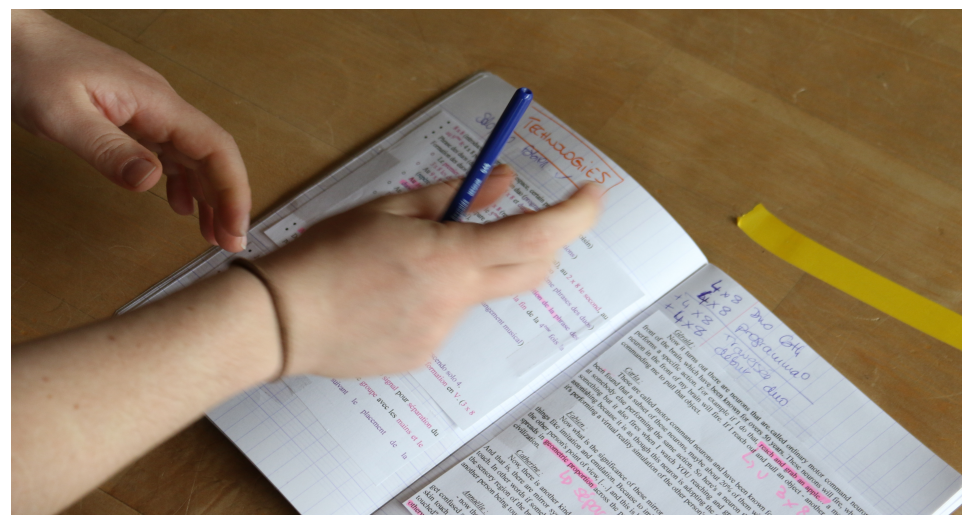

Fig. 9. D12 annotated and integrated part of the rehearsal notebook in her personal notebook.

$\mathrm{RD}$ described that the goal of using compiling platforms is to disseminate the same information among all individuals. Although the learning artifacts are personal and thus sometimes difficult to access by others, compiling them serve to establish common memory, and structure the piece for the group.

5.3.3 MoveOn facilitates dancers' access to videos. Similarly, the participants used digital platforms to regroup and share dance videos. While outside the dance studio, CD shared most of the videos with an online video platform (Vimeo), inside the dance studio, the participants used MoveOn to upload and gather their videos. Both Vimeo and MoveOn served to compile all videos in one place. Additionally, MoveOn stored a trace of the learning pathways of the dancers: how they segmented the videos, how many repetitions they needed for each segment, the speed in which they displayed it and their personal annotations of it. Therefore, through the tool, dancers could come back to their rehearsal strategy simply by playing their segments one after the other. Thus MoveOn served to compile, store and share rehearsals' videos and augmenting them by saving a trace of the learning schedules of each dancer. According to RD, these compilation platforms unify the learning tools used by the group:

It unifies people. It's individual needs, but the more you share them, the more you allow people to be in unison. (RD) 


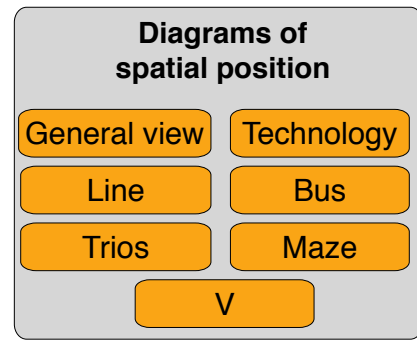

Artifacts

Compilation platforms
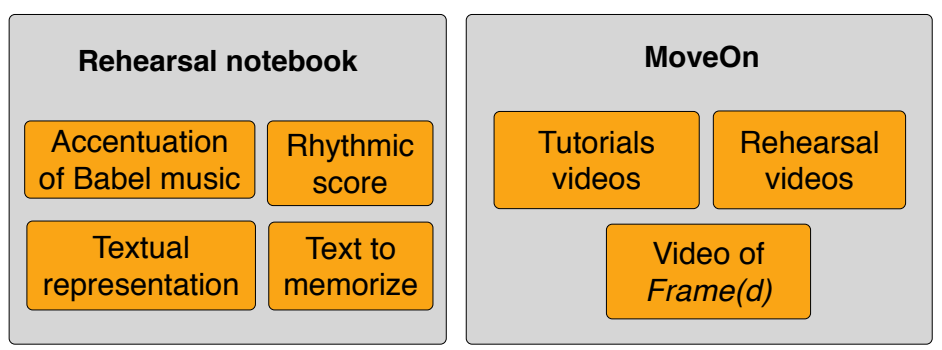

Benesh

Notations

\section{Personal notes}

Fig. 10. This schema depicts the different artifacts (orange rectangles) and their corresponding compilation platforms (grey rectangles). The compilation platforms group artifacts together and play a central role in knowledge sharing. Benesh notations and personal notes are artifacts not compiled in compilation platforms.

\subsection{Overcoming challenges of sharing artifacts through appropriation and distribution of expertise}

Dancers appropriate artifacts to overcome the challenges related to their access. Moreover, the group distribute the tasks according to new expertise that emerged among the group.

5.4.1 Dancers' appropriation of artifacts over time. We observed how dancers appropriate artifacts throughout the process. This is the case with the initial video of Frame(d) provided by the Eastman company to the dancers. This video was originally made for the promotion of the piece, but the dancers used it as a reference point to learn $\operatorname{Frame}(d)$, while RD relied on it to clarify movements. Another example is the videos of the public performances of the piece shot by $\mathrm{CD}$. According to $\mathrm{CD}$, these videos initially helped to archive $\operatorname{Frame}(d)$, to promote the piece and to run fundraising campaigns for future projects. However, the dancers re-purposed them to improve, reflect, and criticize their performance.

Other physical artifacts also shifted from their initial purposes. For example, D5's diagrams became useful before each public performance to place colored tape that represents landmarks on the stage. Benesh notation is another example of how physical artifacts shifted function. Initially, Benesh notation was made to safeguard the piece. However, in the learning process of Frame (d), the Benesh notators used these scores to clarify aspects of the dance to the rest of the group and give them feedback on their performance:

D11: There is a moment when having a notator in a group brought something into the discussions. For example, when $R D$ was teaching something, there were times when D4 said it differently, counted it differently, specified it differently, or said it in another way that was sometimes easier to understand. The fact that a notator has the ability to analyze the movement helps a lot.

5.4.2 Distribution of roles according to dancers' expertise. According to $\mathrm{CD}$, the dancers progressively mastered different aspects of the dance that are complementary. For example, D5 mastered the space and the representation of the scene, D11 and D9 embodied the rhythm precisely, and D4 and D12 were good at analyzing and explaining movements. Due to their expertise, the group considered them as referents. Therefore, they acted as teachers. They corrected other dancers and provided precision on the aspects of the dance that they mastered. D2 explained that "In the end, 
we know there is one dancer who truly knows one part of the piece. The text for you, the rhythm for D9. We shot a film of you, and we used you as the basis for any questions we might have. Almost a referent on each part..." (D2).

We observed that such direct communication replaced the use of the rehearsal notebook at a later stage of the rehearsals: "I haven't used my notebook since... I know where it is, in a little closet, but I prefer to rely on other dancers" (D11). According to D1, the referent dancers roles held a "collective kinesthetic memory" that is key in the group's learning, and that is "not visible in any document" (D1).

We also observed that at a later stage of rehearsals, dancers relied on the referent dancers instead of $\mathrm{RD}$ to learn the dance. $\mathrm{RD}$ 's role then shifted to that of the choreographer, giving instructions on how to perform the piece (intensity, quality of movement), rather than teaching the movement itself.

In addition to looking at referent dancers (D5, D2, D4, D9, D10), the group asked them to generate video tutorials zooming into specific aspects of the choreography. For example, RD filmed D4 performing part 4 of the piece, in close-up, counting the counts. Similarly, RD filmed R10 performing the third part of the piece. These videos tutorials allowed the group to "rely on a good dancer to learn the difficult dance phrases" (D5).

\section{DISCUSSION}

Our findings emphasize how heterogeneous and complementary artifacts that dancers create, appropriate and share, are key for the collective task of learning dance.

\subsection{Providing analytical tools for dancers}

We found that, in a re-staging task, the participants produced an ensemble of artifacts that serve to analyze the dance by decomposing, simplifying and isolating movement, and focusing on different aspects of the choreography. Similarly, MoveOn was exclusively used to segment and thus simplify the dances where knowledge was lacking. Our results show that the artifacts that served to analyze in-depth elements of the choreography became external representations that build "scaffolding for thought", as Kirsh termed [31], which appeared to be a fundamental part of the learning process. This emphasizes that learning dance is not only driven by physical training but also by the ability to analyze the underlying choreographic ideas (space, the position of the dancers, rhythm, etc.).

We found that most of the physical artifacts produced by the participants were used to extract, visualize, and focus on these choreographic elements. For example, D4 produced a musical score (Figure 5.a) to study and memorize the rhythm independently from the rest of the choreography. Thanks to such an external representation of the rhythm, the choreographic structure became clearer and more visible to the learners [31].

The use of artifacts to visualize choreographic structures has been observed by Ciolfi and colleagues [14] in their study of choreographic writing. The authors observed that choreographers generate what they call "choreographic objects" that embody their choreographic ideas. These objects are heterogeneous and can be represented by drawings, texts, diagrams, or videos [16]. These representations structure the piece and allow its transmission to the dancers. This echoes our findings where the artifacts created served to make "choreographic objects" visible, for example the position and relationships between the dancers, the timing, or the rhythm.

- Implication 1: Designing interactive systems for dance learning should facilitate movement analysis in addition to physical practice (which is the focus of most of the existing systems). 


\subsection{Accounting for the diversity of learning methods}

We showed in our study, that challenges around the readability and accessibility of artifacts are due to the fact that they highly embody their creators' perspective, expertise, and personal vocabularies. Participants overcame these challenges by compiling artifacts to share them with each other and build common understanding of the dance.

Our results illustrated how the same choreographic ideas were represented with different artifacts and expressed with different forms and languages depending on the perspectives and expertise of their creators. While some participants preferred videos, others memorized better with text or diagrams. We showed the different ways dancers described the dance in text, with diagrams and annotation, or using MoveOn. Such diversity in the learning tools can be related to the learning style model of Felder et al. [22]. According to this model, there are multiple types of learners, in particular visual and verbal learners. Visual learners tend to remember best when they look at something while verbal learners are more comfortable with written and spoken explanations. However, we showed that such diversity, while preserving personal styles, limits how information is accessible to others that do not share the same learning style, expertise, or language.

To overcome these challenges, technology to support dance learning should provide diverse representations of information including position in space, rhythm, or movements. In addition to that, technology should allow users to select and navigate in the different visualizations available depending on their preferences. An example of such a system is the interactive website Synchronous Objects [37] by William Forsythe that documents his piece One flat thing reproduced by revealing the deep structures of the choreographic ideas (information) through a collection of interactive visualizations that can display movement, rhythm, tempo, etc.

We can go even further by imagining systems that would allow users to interact with diverse tools, and languages that embed their learning preferences.

We suggest that reification can be an appropriate principle to support this. Reification is a design principle within the instrumental interaction model proposed by Beaudouin-Lafon [3], which consists of making an element of the choreography visible and persistent, by reifying it into an interactive object. By reifying different choreographic ideas into a multiplicity of tools, a designer can provide the users with the possibility to navigate between different forms and representations of the same information. MoveOn reifies dancers' personal ways of segmenting and labeling movement. By doing so, the tool accounts for the different focuses and languages that dancers use to characterize movement while offering them a versatile interactive tool that facilitates segmentation and annotation tasks in learning.

- Implication 2: Designing interactive systems for dance learning should account for the diversity of the learning methods by providing the ability to represent the same information through different forms. This could be facilitated by the principle of reification in instrumental interaction.

\subsection{Celebrating shifts in roles and artifacts}

We found that in the process of learning $\operatorname{Frame}(d)$, some dancers shifted roles and became references for the group. This echoes the findings of Hsueh et al. [26] in their study of the creative process in dance. They showed that the roles of the performers and creators alternate between author, curator, planner, and interpreter, creator and improviser. They also showed that the interactions with artifacts during the creative process shifted fluidly between sculpting, layering, and remixing. They call these shifts "slippages" and argue that taking them into account in designing creativity support tools drive creativity forward by opening up pathways into the future. Our results illustrate the importance of these "slippages" of roles and interactions in the context of dance learning. We 
showed how dancers re-appropriated various documents and artifacts and integrated them into an ecology of tools that support their own personal learning journey. We also showed that roles are re-defined throughout the process, resulting in a highly collaborative and continually renewed learning process.

Our results show that throughout the learning process, the expertise of dancers evolves over time. While the artifacts the dancers produce embed their expertise, vocabulary and perspectives, they are in most cases static objects that do not evolve over time. This is why some artifacts are mostly used in the early stages of the process while others appear later on. This is also why some artifacts are abandoned while others are re-appropriated. Through the ecology that the participants compiled, it was possible for them to dynamically select, discard, introduce and re-purpose the artifacts according to their individual and collective needs. This emphasizes the dynamic quality of artifact ecologies as stated by Bødker and Klokmose [6].

From a methodological point of you, it is clear that a longitudinal study was necessary to allow us to fully understand the breadth of the participants practices and how it evolve over time [6]. We acquired a deep understanding of how artifact ecologies mediate collaboration in dance through this longitudinal study. And such methodology, results, and insights apply to other contexts including sports or musical training.

- Implication 3: Designing interactive systems for dance learning should support the diversity of dancers' roles among learners, referents, or choreographers.

\subsection{Supporting distributed expertise among the group}

The phenomenon of a dancer becoming a teacher has already been reported by contemporary dancers to be a factor that positively impacts long-term memory [49]. This phenomenon requires the dancer to acquire a level of expertise before being able to teach a piece. Brown et al. [8] explain that because expertise is distributed among a group of learners, each student specializes in one aspect. Our study of the group's learning process of Frame(d), illustrated how some dancers built expertise in the rhythm, while others preferred spatial positioning. The group then took advantage of such diversity in expertise that altogether became complementary to teach all aspects of the dance. Our results also suggest that the artifacts served to build their creator's expertise. For example, D5 mastered the spatial aspect of $\operatorname{Frame}(d)$ by creating his spacial diagrams.

- Implication 4: Technology supporting dance learning should build on the different expertise of the dancers to foster distributed knowledge among the group.

\section{CONCLUSION}

In this research, we ran a longitudinal study over the course of one year, where we investigated the use of digital and physical artifacts in the re-staging of a dance choreography from the contemporary dance repertoire.

We used artifacts as a lens into the collaborative practice of dance re-staging, to understand how dancers collaborate and how artifacts mediate this collaboration, and we showed that the participants produced and shared several heterogeneous artifacts. We showed how they used these artifacts to analyze, decompose, simplify, isolate, and focus on different aspects of the choreography. While videos and MoveOn were useful tools for participants to focus on movement, the dancers also produced a rich ecology of physical artifacts such as paper, notations, and diagrams.

Such diversity of artifacts allowed us to account for the learning preferences of all dancers. However, sharing these artifacts was challenging because they embody their creator's idiosyncratic perspective, expertise, and language. We showed how compiling these artifacts enabled better sharing and access which supported the building of common knowledge among all learners. 
To conclude, our study shows that creating an ecology of artifacts played an important role in the re-staging of Frame $(d)$. Our results suggest that in addition to physical training, dance learning requires a complex set of cognitive skills consisting of analyzing and understanding the choreography. Therefore, future work should explore avenues for designing tools that support dance learning by making choreographic ideas visual, tangible and interactive.

\section{ACKNOWLEDGMENTS}

We thank all of the dancers involved in this study. This work was partially supported by European Research Council (ERC) grants № 321135 ”CREATIV: Creating Co-Adaptive Human-Computer Partnerships"; the Agence National de la Recherche (ANR) grants № ANR-18-CE33-0002 ”ELEMENT: Enabling Learnability in Embodied Movement Interaction"; and the Diagonale de Paris-Saclay for their involvement.

\section{REFERENCES}

[1] Fraser Anderson, Tovi Grossman, Justin Matejka, and George Fitzmaurice. 2013. YouMove: enhancing movement training with an augmented reality mirror. In Proceedings of the 26th annual ACM symposium on User interface software and technology. ACM, 311-320.

[2] Andreas Aristidou, Efstathios Stavrakis, and Yiorgos Chrysanthou. 2014. Motion Analysis for Folk Dance Evaluation. In GCH. Darmstadt, 55-64.

[3] Michel Beaudouin-Lafon. 2000. Instrumental interaction: an interaction model for designing post-WIMP user interfaces. In Proceedings of the SIGCHI conference on Human Factors in Computing Systems. 446-453.

[4] Bettina Bläsing, Beatriz Calvo-Merino, Emily S Cross, Corinne Jola, Juliane Honisch, and Catherine J Stevens. 2012. Neurocognitive control in dance perception and performance. Acta psychologica 139, 2 (2012), 300-308.

[5] Bettina Bläsing, Martin Puttke, and Thomas Schack. 2010. The neurocognition of dance. London: Psychology Press.

[6] Susanne Bødker and Clemens Nylandsted Klokmose. 2012. Dynamics in artifact ecologies. In Proceedings of the 7th Nordic Conference on Human-Computer Interaction: Making Sense Through Design. 448-457.

[7] Virginia Braun and Victoria Clarke. 2006. Using thematic analysis in psychology. Qualitative research in psychology 3 , 2 (2006), 77-101.

[8] Ann L Brown, Doris Ash, Martha Rutherford, Kathryn Nakagawa, Ann Gordon, and Joseph C Campione. 1993. Distributed expertise in the classroom. Distributed cognitions: Psychological and educational considerations (1993), 188-228.

[9] Diogo Cabral, Urândia Carvalho, João Silva, João Valente, Carla Fernandes, and Nuno Correia. 2011. Multimodal video annotation for contemporary dance creation. In CHI'11 Extended Abstracts on Human Factors in Computing Systems 2293-2298.

[10] Carla Carmona. 2018. Dance and Embodied Cognition: Motivations for the Enactivist Program. Rivista Italiana di Filosofia del Linguaggio 12, 2 (2018).

[11] Erin A. Carroll, Danielle Lottridge, Celine Latulipe, Vikash Singh, and Melissa Word. 2012. Bodies in Critique: A Technological Intervention in the Dance Production Process. In Proceedings of the ACM 2012 Conference on Computer Supported Cooperative Work (Seattle, Washington, USA) (CSCW '12). Association for Computing Machinery, New York, NY, USA, 705-714. https://doi.org/10.1145/2145204.2145311

[12] Jacky CP Chan, Howard Leung, Jeff KT Tang, and Taku Komura. 2010. A virtual reality dance training system using motion capture technology. IEEE Transactions on Learning Technologies 4, 2 (2010), 187-195.

[13] Marianela Ciolfi Felice. 2018. Supporting expert creative practice. Ph.D. Dissertation. Paris Saclay.

[14] Marianela Ciolfi Felice, Sarah Fdili Alaoui, and Wendy E Mackay. 2016. How do choreographers craft dance? Designing for a choreographer-technology partnership. In Proceedings of the 3rd International Symposium on Movement and Computing. 1-8.

[15] Marianela Ciolfi Felice, Sarah Fdili Alaoui, and Wendy E Mackay. 2018. Knotation: exploring and documenting choreographic processes. In Proceedings of the 2018 CHI Conference on Human Factors in Computing Systems. 1-12.

[16] Scott Delahunta, Wayne McGregor, and Alan Blackwell. 2004. Transactables. Performance Research 9, 2 (2004), 67-72.

[17] Scott Delahunta and Norah Zuniga Shaw. 2008. Choreographic resources agents, archives, scores and installations. Performance Research 13, 1 (2008), 131-133.

[18] Scott Delahunta and Norah Zuniga Shaw. 2006. Constructing memories: Creation of the choreographic resource. Performance Research 11, 4 (2006), 53-62. 
[19] Augusto Dias Pereira dos Santos, Lian Loke, and Roberto Martinez-Maldonado. 2018. Exploring Video Annotation as a Tool to Support Dance Teaching. In Proceedings of the 30th Australian Conference on Computer-Human Interaction (Melbourne, Australia) (OzCHI '18). Association for Computing Machinery, New York, NY, USA, 448-452. https: //doi.org/10.1145/3292147.3292194

[20] Katerina El Raheb, Aristotelis Kasomoulis, Akrivi Katifori, Marianna Rezkalla, and Yannis Ioannidis. 2018. A Web-Based System for Annotation of Dance Multimodal Recordings by Dance Practitioners and Experts. In Proceedings of the 5th International Conference on Movement and Computing (Genoa, Italy) (MOCO '18). Association for Computing Machinery, New York, NY, USA, Article 8, 8 pages. https://doi.org/10.1145/3212721.3212722

[21] K Anders Ericsson, Ralf T Krampe, and Clemens Tesch-Römer. 1993. The role of deliberate practice in the acquisition of expert performance. Psychological review 100, 3 (1993), 363.

[22] Richard M Felder, Linda K Silverman, et al. 1988. Learning and teaching styles in engineering education. Engineering education 78, 7 (1988), 674-681.

[23] John C Flanagan. 1954. The critical incident technique. Psychological bulletin 51, 4 (1954), 327.

[24] Jérémie Garcia, Theophanis Tsandilas, Carlos Agon, and Wendy E Mackay. 2014. Structured observation with polyphony: a multifaceted tool for studying music composition. In Proceedings of the 2014 conference on Designing interactive systems. 199-208.

[25] James Hollan, Edwin Hutchins, and David Kirsh. 2000. Distributed cognition: toward a new foundation for humancomputer interaction research. ACM Transactions on Computer-Human Interaction (TOCHI) 7, 2 (2000), 174-196.

[26] Stacy Hsueh, Sarah Fdili Alaoui, and Wendy E Mackay. 2019. Deconstructing Creativity: Non-Linear Processes and Fluid Roles in Contemporary Music and Dance. Proceedings of the ACM on Human-Computer Interaction 3, CSCW (2019), 1-21.

[27] Stacy Hsueh, Sarah Fdili Alaoui, and Wendy E Mackay. 2019. Understanding Kinaesthetic Creativity in Dance. In Proceedings of the 2019 CHI Conference on Human Factors in Computing Systems. 1-12.

[28] Edwin Hutchins. 1995. Cognition in the Wild. Number 1995. MIT press.

[29] Edwin Hutchins. 2014. The technology of team navigation. In Intellectual teamwork. Psychology Press, 205-234.

[30] Heekyoung Jung, Erik Stolterman, Will Ryan, Tonya Thompson, and Marty Siegel. 2008. Toward a Framework for Ecologies of Artifacts: How Are Digital Artifacts Interconnected within a Personal Life?. In Proceedings of the 5th Nordic Conference on Human-Computer Interaction: Building Bridges (Lund, Sweden) (NordiCHI '08). Association for Computing Machinery, New York, NY, USA, 201-210. https://doi.org/10.1145/1463160.1463182

[31] David Kirsh. 2010. Thinking with external representations. AI \& society 25, 4 (2010), 441-454.

[32] David Kirsh, Dafne Muntanyola, R Joanne Jao, Amy Lew, and Matt Sugihara. 2009. Choreographic methods for creating novel, high quality dance. In Proceedings, DESFORM 5th international workshop on design \& semantics \& form. 188-195.

[33] Seymour Kleinman. 1975. Movement notation systems: An introduction. Quest 23, 1 (1975), 33-34.

[34] Z Li. 2011. How technology shapes our way of teaching dance. Proceedings of the 2010 World Dance Alliance Global Summit (1), Linda Caldwell (Ed.) 1 (2011).

[35] Wendy E Mackay. 2002. Using video to support interaction design. DVD Tutorial, CHI 2, 5 (2002).

[36] Martin Nielsen and Susanne Bødker. 2004. Desktop Computing-Distributed Cognition in a Tax Office. Australasian Journal of Information Systems 11, 2 (2004).

[37] Maria Palazzi, Norah Zuniga Shaw, William Forsythe, Matthew Lewis, Beth Albright, Michael Andereck, Sucheta Bhatawadekar, Hyowon Ban, Andrew Calhoun, Jane Drozd, et al. 2009. Synchronous objects for one flat thing, reproduced. In ACM SIGGRAPH 2009 Art Gallery. 1-1.

[38] Katerina El Raheb, Marina Stergiou, Akrivi Katifori, and Yannis Ioannidis. 2019. Dance Interactive Learning Systems: A Study on Interaction Workflow and Teaching Approaches. ACM Comput. Surv. 52, 3, Article 50 (June 2019 ), 37 pages. https://doi.org/10.1145/3323335

[39] Jean-Philippe Rivière, Sarah Fdili Alaoui, Baptiste Caramiaux, and Wendy E Mackay. 2019. Capturing Movement Decomposition to Support Learning and Teaching in Contemporary Dance. Proceedings of the ACM on Human-Computer Interaction 3, CSCW (2019), 86.

[40] Yvonne Rogers. 2011. Interaction design gone wild: striving for wild theory. interactions 18, 4 (2011), 58-62.

[41] Gavriel Salomon. 1997. Distributed cognitions: Psychological and educational considerations. Cambridge University Press.

[42] R Keith Sawyer. 2005. The Cambridge handbook of the learning sciences. Cambridge University Press.

[43] Vikash Singh, Celine Latulipe, Erin Carroll, and Danielle Lottridge. 2011. The Choreographer's Notebook: A Video Annotation System for Dancers and Choreographers. In Proceedings of the 8th ACM Conference on Creativity and Cognition (Atlanta, Georgia, USA) (C\&C '11). Association for Computing Machinery, New York, NY, USA, 197-206. https://doi.org/10.1145/2069618.2069653

[44] Miriam Solomon. 2007. Situated cognition. In Philosophy of psychology and cognitive science. Elsevier, 413-428.

[45] Gerry Stahl. 2004. Building collaborative knowing. In What we know about CSCL. Springer, 53-85. 
[46] Gerry Stahl. 2013. Theories of cognition in collaborative learning. In The international handbook of collaborative learning. Routledge, 86-102.

[47] Gerry Stahl, S Ludvigsen, Nancy Law, and Ulrike Cress. 2014. CSCL artifacts. International fournal of ComputerSupported Collaborative Learning 9, 3 (2014), 237-245.

[48] Catherine Stevens et al. 2005. 13. Chronology of Creating A Dance: Anna Smith's Red Rain. Thinking in Four Dimensions: Creativity and Cognition in Contemporary Dance (2005), 169.

[49] Catherine J Stevens, Kim Vincs, Scott Delahunta, and Elizabeth Old. 2019. Long-term memory for contemporary dance is distributed and collaborative. Acta psychologica 194 (2019), 17-27.

[50] Francisco J Varela, Evan Thompson, and Eleanor Rosch. 2016. The embodied mind: Cognitive science and human experience. MIT press.

[51] Dhaval Vyas and Alan Dix. 2007. Artefact Ecologies: Supporting Embodied Meeting Practices with Distance Access. Proceedings of UbiComp 2007 (2007), 117-122.

Received December 2020; revised January 2021; accepted February 2021 